\title{
Decolonization of Methicillin resistant Staphylococcus aureus: Role in the Neonatal Intensive Care Unit
}

\author{
Ji Won Koh, M.D., In Gyu Song, M.D., Sae Yun Kim, M.D., Young Hwa Jung, M.D., Seung Han Shin, M.D., Ee- \\ Kyung Kim, M.D., Han-Suk Kim, M.D., Jung-Hwan Choi, M.D., and Ju-Young Lee, M.D.* \\ Department of Pediatrics, Seoul National University College of Medicine, Seoul, Korea \\ Department of Pediatrics*, Inha University College of Medicine, Incheon, Korea
}

\section{ABSTRACT}

Purpose: We aimed to assess the incidence of methicillin-resistant Staphylococcus aureus (MRSA) colonization and infection in a neonatal intensive care unit (NICU) by using various decolonization methods and to evaluate their efficacy.

Methods: Medical records of all neonates who were admitted to the NICU of Seoul National University Children's Hospital were retrospectively reviewed. Surveillance culture were obtained for all neonates in the NICU 48 hours after admission. Three periods with different decolonization methods were compared; Period 1 was without any decolonization measures (July 1, 2009 to August 26, 2010). In period 2, intranasal mupirocin and chlorhexidine gluconate bathing were administered to MRSA-colonized neonates (August 27, 2010 to September 6, 2011). In period 3, only chlorhexidine bathing was performed for MRSA-colonized infants (September 7, 2011 to August 31, 2012).

Results: A total of 1,378 infants were admitted to the NICU during the study period. Baseline demographic and clinical characteristics were similar among the 3 periods. The incidence of MRSA colonization per 1,000 patient-days was 6.27 for period 1, 7.02 for period 2, and 6.29 for period 3; however, these values were not significantly different. The incidence of MRSA infection was highest in period 3, with 0.69 cases per 1,000 patient-days; however, this finding was not significant. The MRSA infection/ colonization ratio also did not differ significantly among the 3 study periods.

Conclusion: Decolonization of MRSA in the NICU with the application of chlorhexidine gluconate bathing alone or in combination with intranasal mupirocin were not effective in decreasing the incidence of MRSA colonization and infection.

Key Words: Methicillin-resistant Staphylococcus aureus, Colonization, Mupirocin, Infection

Received: 10 February 2016

Revised: 4 April 2016

Accepted: 6 April 2016

Correspondence to:

\section{Han-Suk Kim}

Department of Pediatrics, Seoul National University College of Medicine, 101 Daehak-ro, Jongnogu, Seoul 03082, Korea

Tel: +82-2-2072-3553

Fax: +82-2-2072-0590

E-mail: kimhans@snu.ac.kr

Copyright(c)

By Korean Society of Neonatology. All right reserved.

This is an Open-Access article distributed under the terms of the Creative Commons Attribution Non-Commercial License (http://creativecommons.org/licenses/ by-nc/4.0), which permits unrestricted non-commercial use, distribution, and reproduction in any medium, provided the original work is properly cited. 


\section{INTRODUCTION}

Methicillin-resistant Staphylococcus aureus (MRSA) has become increasingly prevalent in most parts of the world, and is a major pathogen of bacteremia in neonatal intensive care units (NICUs) $^{1-3)}$.

The National Nosocomial Infections Surveillance System observed more than a $300 \%$ increase in the incidence of lateonset MRSA infections in NICUs between 1995 and 2004 ${ }^{4}$. In a recent meta-analysis, the prevalence of MRSA colonization upon admission was $1.5 \%$ (95\% confidence interval [CI] 0.92.2) among NICU patients ${ }^{5}$. Several factors contribute to NICU patients being at a particularly high risk of being colonized and infected with MRSA, including an immature immune system, exposure to numerous invasive procedures ${ }^{6}$, , prolonged hospitalization ${ }^{7}$, and, especially, a high frequency of contact with health care providers ${ }^{8,9)}$.

It has been reported that MRSA colonization or infection is associated with mortality in the NICU ${ }^{10)}$. Because MRSA colonization itself has a relative risk of 24.2 (95\% CI 8.9-66.0) to develop a MRSA infection, prevention of MRSA transmission in the NICU is important ${ }^{5,11)}$. Therefore, individual NICUs have adopted various combinations of surveillance, special precautions and decolonization strategies to minimize MRSA infections $^{7,12-14)}$. For decolonization, the application of topical intranasal mupirocin with/without chlorhexidine showers has been assessed most extensively. In a recent report by Huang et al., $4.2 \%$ of hospitalized infants had MRSA colonization, where mupirocin treatment was effective in decolonizing MRSA carriage and reducing subsequent MRSA infection ${ }^{15)}$.

Although numerous methods regarding attempts to eradicate MRSA outbreaks in NICUs have been published, a consensus for guidelines is still lacking. Gerber et al. ${ }^{16)}$ published a consensus statement in 2006 along with guidelines for the prevention and control of MRSA colonization and infection. Additionally, mupirocin was recommended for the decolonization of infants or healthcare workers if deemed necessary by the institution.

Therefore, in this study, by using chlorhexidine gluconate bathing alone or in combination with intranasal mupirocin, we aimed to compare the incidence of MRSA colonization and infection and evaluate the efficacy of these decolonization methods.

\section{MATERIALS AND METHODS}

\section{Study Poplation}

This retrospective cohort study was conducted from July 2009 to August 2012 in the NICU at the Seoul National University Children's Hospital (SNUCH) which houses a 40-bed, level III NICU. During the study period, an average of, 474.3 NICU admissions per year was observed, and among them, 113.7 (24\%) admissions per year were infants with a very low birth weight $(<1,500 \mathrm{~g})$.

\section{Definitions}

Colonization was defined as the isolation of MRSA from the anterior nares, rectum, and axillary without evidence of infection, whereas infection was defined as the isolation of MRSA from normally sterile sites including blood, urine, and cerebrospinal fluid, or from non-sterile sites (e.g., skin, eye, or umbilical stump), with the presence of clinical signs of infection using the National Healthcare Safety Network criteria for nosocomial infection as a guide ${ }^{17)}$. However, in this study, we used the term 'infection' to denote a blood stream infection.

An incidence case was defined as a surveillance culture or clinical culture positive for MRSA from a sample obtained more than 48 hours after admission with no previous history of MRSA colonization or infection.

\section{Infection Control and Prevention Program}

Surveillance cultures were obtained for all inborn and outborn neonates in the NICU 48 hours after admission. A total of 1,378 neonates were screened during the study period without any missing data. Weekly surveillance cultures were obtained in MRSA-colonized or infected neonates during their stay in the NICU. Culture samples were obtained by swabbing the following 3 areas: the anterior nares, rectum, and axilla.

Cohort isolation was initiated if an infant had a laboratory proof of colonization or infection with MRSA. Visible signs were placed on the beds of colonized or infected patients as a reminder of the need for contact precautions such as gowning, gloving and hand washing. No culturing was performed for environmental samples. An infection control nurse instructed health care staff on the proper contact isolation techniques.

Colonized patients confirmed by nasal swab cultures received mupirocin ointment twice daily to the anterior nares for 7 days. 
Patients who were colonized with MRSA in the axilla received a $0.4 \%$ chlorhexidine bath for 3 subsequent days. All patients with known MRSA colonization underwent repeated surveillance culturing of the samples on a weekly basis after receiving decolonization treatment to document clearance of MRSA and to identify persistent MRSA carriage. Decolonization treatments were continued until 2 sets of post-treatment MRSA surveillance cultures were found to be negative.

\section{Study Design and Data Collection}

After approval from the institutional review board of the hospital, we retrospectively reviewed medical records for any MRSA positive surveillance culture samples or MRSA infection cases during the study periods. The following variables were collected: birth weight, gestational age, sex, mode of delivery, clinical conditions at the time when a culture was found to be positive, and the use of central catheters.

We compared 3 time periods to evaluate the impact of 2 different decolonization interventions on neonates with MRSA positive, who were admitted to the SNUCH from July 2009 to August 2012. Baseline observations were made during the 13month period from July 1, 2009 to August 26, 2010 without any decolonization measures (Period 1). Only contact precaution techniques were encouraged. Because various strategies had been newly proposed for MRSA decolonization, 2 interventions were in place during the 24-month period from August 27, 2010 to September 6, 2011 (Period 2) and September 7, 2011 to August 31, 2012 (Period 3). Patients identified as MRSA carriers were routinely treated with intranasal mupirocin and daily bathing with chlorhexidine during period 2 , and chlorhexidine bathing alone was performed in period 3. Baseline characteristics such as parenteral feeding, use of mechanical ventilation, and use of a central venous catheter were compared among the periods.

Other known related factors for MRSA prevention or infection such as probiotics ${ }^{18)}$ and $\mathrm{H}_{2}$-blocker use ${ }^{19)}$, were not studied because we did not routinely use them in the NICU. Breastfeeding ${ }^{20)}$ was also excluded because we did not exclusively feed breast milk to the neonates in the NICU.

The incidence of MRSA colonization or infection per 1,000 patient-days was calculated for each period, and the results were compared.

\section{Statistical Analysis}

Statistical analysis was performed using the SAS system, version 9.2 (SAS institute, Cary, NC, USA). To determine the relationship between continuous variables, a 2-sample t-test was used. If the distribution of the continuous variable did not fit a normal distribution, the Kruskal-Wallis test was used for the analysis. Categorical data were compared by using the $\chi^{2}$ test or the Fisher's exact test. $P$-values less than 0.05 were considered statistically significant for all comparisons.

The rate of S. aureus colonization or infection was calculated as the number of S. aureus infected neonates or colonized neonates per 1,000 patient-days. A comparison of MRSA incidence rates for each period was performed using a generalized linear model.

The statistical analysis of this study was conducted in the Medical Research Collaborating Center of Seoul National University, Seoul, Korea.

\section{RESULTS}

A total of 1,378 infants were admitted to the NICU during the study period. The number of admission cases for each period was 421 cases for period 1, 516 cases for period 2, and 486 cases for period 3. The clinical characteristics of the infants in each time period were compared (Table 1). There were no significant differences among the time periods in regards to the proportion of infants with a gestational age younger than 26 weeks and the number of extremely low birth weight infants (birth weight < $1,000 \mathrm{~g})$. Other baseline demographic and clinical characteristics were similar among the 3 groups, except for the length of stay in the NICU $(P=0.019)$, the number of infants with central catheter use $(P=0.032)$, and the number of infants with the use of a peripherally inserted central catheter $(P=0.009$, Table 1$)$.

The clinical characteristics of MRSA-colonized and MRSAinfected infants are shown in Table 2. There were no significant differences among the groups in regards to the clinical status at the time of the first positive MRSA culture, such as the age at colonization or infection, status of parenteral feeding, mechanical ventilation, and central venous catheter use. The days from admission to colonization or infection were not significantly different among the study periods.

The incidence of MRSA colonization per 1,000 patient-days was 6.27 for period 1, 7.02 for period 2, 6.29 for period 3, and there were no significant differences among the time periods (Table 3). The incidence of MRSA infection was highest in period 3 , with 0.69 cases per 1,000 patient-days; however, this finding 
Table 1. Comparison of Clinical Characteristics of Neonates in Each Period

\begin{tabular}{|c|c|c|c|c|}
\hline & Period 1 & Period 2 & Period 3 & $P$-value \\
\hline No. of admission & 421 & 516 & 486 & \\
\hline GA < 26 weeks, n (\%) & $29(7.1)$ & $24(4.7)$ & $20(4.3)$ & 0.117 \\
\hline GA < 31 weeks, n (\%) & $105(25.6)$ & $107(21.1)$ & $84(18.2)$ & 0.790 \\
\hline BW <1,000 g, n (\%) & $71(17.3)$ & $67(13.2)$ & $49(10.7)$ & 0.192 \\
\hline BW <1,500 g, n (\%) & $116(28.2)$ & $124(24.5)$ & $101(22.0)$ & 0.746 \\
\hline Male:Female (\%) & $53: 37$ & $54: 46$ & $57: 43$ & 0.376 \\
\hline Inborn:Outborn (\%) & $65.2: 34: 8$ & $78.5: 21.5$ & 77.4: 22.6 & $0.001^{*}$ \\
\hline ICU days $^{\dagger}$ (min, max) & $29.2 \pm 38.7(2,264)$ & $25.1 \pm 30.3(2,288)$ & $23.9 \pm 42.1(2,460)$ & $0.019^{*}$ \\
\hline No. of central catheter use & 66 & 93 & 59 & $0.032^{*}$ \\
\hline No. of PICC use & 180 & 246 & 173 & $0.009 *$ \\
\hline
\end{tabular}

Plus-minus values are mean \pm SD.

${ }^{*} P$-value $<0.05$

${ }^{\dagger}$ Minimum and maximum in parenthesis.

Abbreviations: No, number; GA, gestational age; BW, birth weight; ICU, intensive care unit; PICC, peripherally inserted central catheter.

Table 2. Clinical Characteristics of Colonized and Infected Infants

\begin{tabular}{|c|c|c|c|c|}
\hline & Period 1 & Period 2 & Period 3 & $P$-value \\
\hline \multicolumn{5}{|l|}{ MRSA colonization } \\
\hline Days to colonization ${ }^{* \dagger}$ & $16 \cdot 0 \pm 29.2(0,145)$ & $14.0 \pm 13.9(0,63)$ & $15.3 \pm 22.8(0,123)$ & 0.137 \\
\hline Age at colonization ${ }^{\dagger}$ & $29.8 \pm 32.1(1,146)$ & $21.5 \pm 18.9(2,126)$ & $31.0 \pm 42.8(0,209)$ & 0.741 \\
\hline \multicolumn{5}{|c|}{ Clinical status at time of first positive culture, $\mathrm{n}(\%)$} \\
\hline Parenteral feeding & $48(62.3)$ & $49(53.9)$ & $48(65.8)$ & 0.270 \\
\hline \multicolumn{5}{|l|}{ Gastric tube use } \\
\hline Mechanical ventilation & $20(26.0)$ & $36(39.6)$ & $19(26.4)$ & 0.095 \\
\hline Central venous line & $4(5.2)$ & $10(11.0)$ & $7(9.6)$ & 0.394 \\
\hline PICC & $31(40.3)$ & $51(56.0)$ & $30(41.1)$ & 0.067 \\
\hline \multicolumn{5}{|l|}{ MRSA infection } \\
\hline Days to infection*' (min, max) & $12.4 \pm 11.8(0,33)$ & $29.6 \pm 20.6(3,65)$ & $29.3 \pm 32.2(3,103)$ & 0.160 \\
\hline Age at infection ${ }^{\dagger}(\min , \max )$ & $25.0 \pm 17.9(3,59)$ & $35.3 \pm 27.5(4,87)$ & $30.0 \pm 31.8(3,103)$ & 0.678 \\
\hline \multicolumn{5}{|c|}{ Clinical status at time of first positive culture, $\mathrm{n}(\%)$} \\
\hline Parenteral feeding & $6(85.7)$ & $4(57.1)$ & $8(100)$ & 0.086 \\
\hline Mechanical ventilation & $3(42.9)$ & $5(71.4)$ & $3(37.5)$ & 0.562 \\
\hline Central venous line & $2(28.6)$ & $2(28.6)$ & $2(25.0)$ & 1.000 \\
\hline PICC & $3(42.9)$ & $3(42.9)$ & $5(62.5)$ & 0.757 \\
\hline
\end{tabular}

Plus-minus values are mean \pm SD.

*Time from NICU admission to first positive surveillance or culture result, in days.

${ }^{\dagger}$ Minimum and maximum in parenthesis.

Abbreviation: MRSA, methicillin-resistant Staphylococcus aureus; PICC, peripherally inserted central catheter.

was not significantly different from the other time periods (Table 3). The infection/colonization ratio for each period did not significantly differ among the periods. Among the 152 inborn MRSA colonized infants, $11.84 \%(n=18)$ developed a MRSA infection. This statistic is comparable to that of outborn infants, which was $4.5 \%(n=4)$ among 89 MRSA-colonized infants.
The overall incidence of MRSA colonization and MRSA infection was $17 \%$ (241 MRSA colonization cases/1,423 total cases) and 1.5\% (22 MRSA infection cases/1,423 total cases), respectively. 
Table 3. Comparison of the Incidence of Methicillin-Resistant Staphylococcus Aureus Colonization and Infection in Each Period

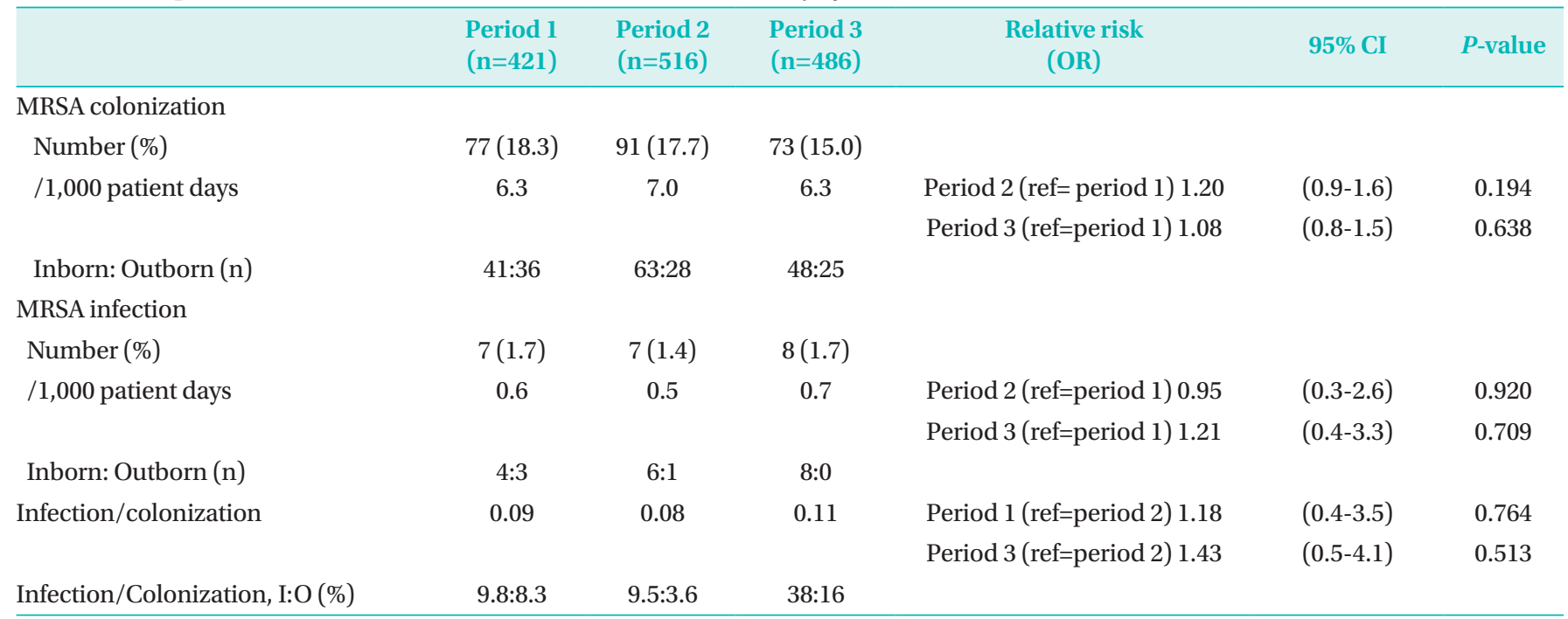

Abbreviations: MRSA, methicillin-resistant Staphylococcus aureus; I, inborn; O, outborn; ref, reference; CI, confidence interval.

\section{DISCUSSION}

The first neonatal case of MRSA infection occurring in the NICU was published in $1981^{21)}$. Since then, MRSA has become a frequent source of infections affecting premature and critically ill neonates in NICUs ${ }^{4,22,23)}$. Approximately $0.6-8.4 \%$ of NICU patients were reported to be colonized or infected with MRSA during the study periods ${ }^{11,14,22)}$. From a study previously published at the SNUCH, S. aureus was proven to be the most common causative organism of culture-proven sepsis for inborn neonates admitted to the NICU between 1996 and $2013^{24)}$. Among the 62 S. aureus infected patients, 59 patients (95.2\%) were MRSA infection based on their antibiotic sensitivity test, which implies that MRSA is the major risk factor for neonatal sepsis in our NICU. In this study, $17 \%$ were found to be MRSAcolonized patients in their first surveillance 48 hours after admission, and $7.5 \%$ of MRSA-colonized neonates developed a MRSA infection, which is also comparable to previous results in which the infection rate ranges from $1.8-26 \%^{11,15,22)}$.

Previous studies have described the various risk factors that predispose infants to MRSA colonization and infection. Khoury et al. ${ }^{25)}$ described that low birth weight and young gestational age were significant risk factors for MRSA colonization and infection. Procedures and devices such as endotracheal intubation and mechanical ventilation ${ }^{6,16,24,26)}$, percutaneous central venous catheterization $^{16)}$, and parenteral feedings ${ }^{16)}$ are also known to be risk factors for MRSA infection. In a recent study, outborn infants were also described as a risk factor for MRSA colonization ${ }^{5)}$. However, inborn infants were dominant for both MRSA colonization and infection in this study.

Because we aimed to evaluate the effectiveness of each decolonization method as an infection prevention measure, we compared the incidence of MRSA colonization, MRSA infection, and the probability of developing a MRSA infection. However, there were no significant differences among the 3 periods. Therefore, the results indicate that MRSA decolonization with chlorhexidine bathing with or without intranasal mupirocin might be ineffective in eradicating MRSA and further preventing MRSA infection in MRSA-colonized patients in the NICU. Loeb and colleagues also concluded that there is insufficient evidence to support the use of topical or systemic antimicrobial therapy for eradicating $\mathrm{MRSA}^{27)}$. They also warned of adverse effects and resistance from using antimicrobial agents ${ }^{27)}$. Another study from 2009 also showed that mupirocin did not help to control MRSA outbreaks, where hand hygiene and appropriate cohort isolation played an important role in decolonizing $\mathrm{MRSA}^{28)}$. In other studies, however, whole-body washing with a $4 \%$ chlorhexidine solution was effective in reducing skin colonization of MRSA ${ }^{29)}$. Additionally, mupirocin topical therapy was shown to prevent MRSA infection ${ }^{15)}$; however, there is a concern regarding the development of resistance; the resistance rate is reported to be approximately $1 \%$ during treatment ${ }^{30}$. Therefore, the appropriate measurement for MRSA eradication is still uncertain ${ }^{31)}$. For 
MRSA infection, vancomycin has been the antibiotic of choice ${ }^{26)}$. After a blood culture sample tested positive, vancomycin was our first choice, which was initiated from the first culture-positive day for 10-21 days.

This study had multiple limitations. First, this study was retrospective and was performed at a single-center NICU; therefore, the results might not be generalizable to all other NICUs. Second, we did not show the data for the decolonization rate of the MRSA-colonized population because of recurrent recolonization. Popoola et al. ${ }^{32)}$ reported that $50 \%$ of neonates staying in the NICU for 21 days or more became recolonized with MRSA. Third, we did not perform the molecular analysis on the MRSA isolates. Molecular analyses have been conducted in many previous studies ${ }^{6,28,33)}$, and they have helped to elucidate trends in the appearance of new strains and in changing antibiotic susceptibility patterns.

Because there are no standardized guidelines for the control of MRSA colonization and infection in NICUs, we need multivariable approaches that include identifying colonized neonates and placing them on proper contact precautions, cohorting, encouraging proper hand hygiene of healthcare workers, environmental cleaning and the decolonization of colonized neonates $^{34-36)}$.

In conclusion, we recommend continuous surveillance to detect MRSA colonization so that appropriate patients can be placed into contact and cohort isolation. Further studies should evaluate and compare strategies to prevent MRSA infection in the NICU to determine optimal decolonization regimens and methods to interrupt reservoirs of transmission.

\section{REFERENCES}

1) Saiman L, Cronquist A, Wu F, Zhou J, Rubenstein D, Eisner W, et al. An outbreak of methicillin-resistant Staphylococcus aureus in a neonatal intensive care unit. Infect Control Hosp Epidemiol 2003;24:317-21.

2) Nambiar S, Herwaldt LA, Singh N. Outbreak of invasive disease caused by methicillin-resistant Staphylococcus aureus in neonates and prevalence in the neonatal intensive care unit. Pediatr Crit Care Med 2003;4:220-26.

3) Köck R, Becker K, Cookson B, van Gemert-Pijnen JE, Harbarth S, Kluytmans J, et al. Methicillin-resistant Staphylococcus aureus (MRSA): burden of disease and control challenges in Europe. Euro Surveill 2010;15:19688.
4) Lessa FC, Edwards JR, Fridkin SK, Tenover FC, Horan TC, Gorwitz RJ. Trends in incidence of late-onset methicillinresistant Staphylococcus aureus infection in neonatal intensive care units: data from National Nosocomial Infections Surveillance System, 1955-2004. Pediatr Infect Dis J 2009;28: 577-81.

5) Zervou FN, Zacharioudakis IM, Ziakas PD, Mylonakis E. MRSA colonization and risk of infection in the neonatal and pediatric ICU: a meta-analysis. Pediatrics 2014;133:e1015-23.

6) Nelson MU, Bizzarro MJ, Baltimore RS, Dembry LM, Gallagher PG. Clinical and molecular epidemiology of methicillinresistant Staphylococcus aureus in a neonatal intensive care unit in the decade following implementation of an active detection and isolation program. J Clin Microbiol 2015;53: 2492-501.

7) Maraqa NF, Aigbivbalu L, Masnita-Iusan C, Wludyka P, Shareef $\mathrm{Z}$, Bailey C, et al. Prevalence of and risk factors for methicillinresistant Staphylococcus aureus colonization and infection among infants at a level III neonatal intensive care unit. Am J Infect Control 2011;39:35-41.

8) Stein M, Navon-Venezia S, Chmelnitsky I, Kohelet D, Schwartz $\mathrm{O}$, Agmon O, et al. An outbreak of new, nonmultidrug-resistant, methicillin-resistant Staphylococcus aureus strain (scc mec type iiia variant-1) in the neonatal intensive care unit transmitted by a staff member. Pediatr Infect Dis J 2006;25: 557-9.

9) Cohen B, Saiman L, Cimiotti J, Larson E. Factors associated with hand hygiene practices in two neonatal intensive care units. Pediatr Infect Dis J 2003;22:494-9.

10) Song $X$, Perencevich E, Campos J, Short BL, Singh N. Clinical and economic impact of methicillin-resistant Staphylococcus aureus colonization or infection on neonates in intensive care units. Infect Control Hosp Epidemiol 2010;31:177-82.

11) Huang YC, Chou YH, Su LH, Lien RI, Lin TY. Methicillinresistant Staphylococcus aureus colonization and its association with infection among infants hospitalized in neonatal intensive care units. Pediatrics 2006;118:469-74.

12) Haley RW, Cushion NB, Tenover FC, Bannerman TL, Dryer D, Ross J, et al. Eradication of endemic methicillin-resistant Staphylococcus aureus infections from a neonatal intensive care unit. J Infect Dis 1995;17:614-24.

13) Back NA, Linnemann CC Jr., Staneck JL, Kotagal UR. Control of methicillin-resistant Staphylococcus aureus in a neonatal intensive care unit: use of intensive microbiologic surveillance and mupirocin. Infect Control Hosp Epidemiol 1996; 17:227-31.

14) Gregory ML, Eichenwald EC, Puopolo KM. Seven-year experience with a surveillance program to reduce methicillinresistant Staphylococcus aureus colonization in a neonatal 
intensive care unit. Pediatrics 2009;123:e790-6.

15) Huang YC, Lien RI, Lin TY. Effect of mupirocin decolonization on subsequent methicillin-resistant Staphylococcus aureus infection in infants in neonatal intensive care units. Pediatr Infect Dis J 2015;34:241-5.

16) Gerber SI, Jones RC, Scott MV, Price JS, Dworkin MS, Filippell $\mathrm{MB}$, et al. Management of outbreaks of methicillin-resistant Staphylococcus aureus infection in the neonatal intensive care unit: a consensus statement. Infect Control Hosp Epidemiol 2006;27:139-45.

17) Edwards JR, Peterson $\mathrm{KD}, \mathrm{Mu} Y$, Banerjee $S$, Allen-Bridson $\mathrm{K}$, Morrell G, et al. National Healthcare Safety Network (NHSN) report: data summary for 2006 through 2008, issued December 2009. Am J Infect Control 2009;37:783-805.

18) Sikorska H, Smoragiewicz W. Role of probiotics in the prevention and treatment of meticillin-resistant Staphylococcus aureus infections. Int J Antimicrob Agents 2013;42:475-81.

19) Atmaca $O$, Zarakolu P, Karahan C, Cakar B, Unal S. Risk factors and antibiotic use in methicillin-resistant Staphylococcus aureus bacteremia in hospitalized patients at Hacettepe University Adult and Oncology Hospitals (2004-2011) and antimicrobial susceptibilities of the isolates: a nested case-control study. Mikrobiyol Bul 2014;48:523-37.

20) Behari P, Englund J, Alcasid G, Garcia-Houchins S, Weber SG. Transmission of methicillin-resistant Staphylococcus aureus to preterm infants through breast milk. Infect Control Hosp Epidemiol 2004;25:778-80.

21) Weeks JL, Garcia-Prats JA, Baker CJ. Methicillin-resistant Staphylococcus aureus osteomyelitis in a neonate. JAMA 1981; 245:1662-4.

22) Carey AJ, Duchon J, Della-Latta P, Saiman L. The epidemiology of methicillin-susceptible and methicillin-resistant Staphylococcus aureus in a neonatal intensive care unit, 2000-2007. J Perinatol 2010;30:135-9.

23) McAdams RM, Ellis MW, Trevino S, Rajnik M. Spread of methicillin-resistant Staphylococcus aureus USA300 in a neonatal intensive care unit. Pediatr Int 2008;50:810-5.

24) Heo JS, Shin SH, Jung YH, Kim EK, Choi EH, Kim HS, et al. Neonatal sepsis in a rapidly growing, tertiary neonatal intensive care unit: trends over 18 years. Pediatr Int 2015;57:909-16.

25) Khoury J, Jones M, Grim A, Dunne WM, Fraser V. Eradication of methicillin-resistant Staphylococcus aureus from a neonatal intensive care unit by active surveillance and aggressive infection control measures. Infect Control Hosp Epidemiol 2005;26:616-21.
26) Kang HC, Lee KC, Kim SS, Park JO, Kim CH. Staphylococcal infection in the neonatal intensive care unit. J Korean Soc Neonatol 2007;14:215-20.

27) Loeb MB, Main C, Eady A, Walker-Dilks C. Antimicrobial drugs for treating methicillin-resistant Staphylococcus aureus colonization. Cochrane Database Syst Rev 2003:Cd003340. doi:10.1002/14651858.cd003340.

28) Lepelletier D, Corvec S, Caillon J, Reynaud A, Rozé JC, GrasLeguen C. Eradication of methicillin-resistant Staphylococcus aureus in a neonatal intensive care unit: which measures for which success? Am J Infect Control 2009;37:195-200.

29) Wendt C, Schinke S, Württemberger M, Oberdorfer K, BockHensley $\mathrm{O}$, von Baum H. Value of whole-body washing with chlorhexidine for the eradication of methicillin-resistant Staphylococcus aureus: a randomized, placebo-controlled, double-blind clinical trial. Infect Control Hosp Epidemiol 2007;28:1036-43.

30) Ammerlaan HS, Kluytmans JA, Wertheim HF, Nouwen JL, Bonten MJ. Eradication of methicillin-resistant Staphylococcus aureus carriage: a systematic review. Clin Infect Dis 2009; 48:922-30.

31) Simor AE. Staphylococcal decolonisation: an effective strategy for prevention of infection? Lancet Infect Dis 2011;11:952-62.

32) Popoola VO, Budd A, Witting SM, Ross T, Aucott SW, Perl TM, et al. Methicillin-resistant Staphylococcus aureus transmission and infections in a neonatal intensive care unit despite active surveillance cultures and decolonization: challenges for infection prevention. Infect Control Hosp Epidemiol 2014;35: 412-8.

33) Machuca MA, Sosa LM, González CI. Molecular typing and virulence characteristic of methicillin-resistant Staphylococcus aureus isolates from pediatric patients in Bucaramanga, Colombia. PLoS One 2013;8:e73434.

34) Fraser TG, Fatica C, Scarpelli M, Arroliga AC, Guzman J, Shrestha NK, et al. Decrease in Staphylococcus aureus colonization and hospital-acquired infection in a medical intensive care unit after institution of an active surveillance and decolonization program. Infect Control Hosp Epidemiol 2010; 31:779-83.

35) Huang SS, Septimus E, Kleinman K, Moody J, Hickok J, Avery $\mathrm{TR}$, et al. Targeted versus universal decolonization to prevent ICU infection. N Engl J Med 2013;368:2255-65.

36) Kim EA. Methicillin-resistant Staphylococcus aureus (MRSA) infection in neonates. Neonatal Med 2013;20:354-60. 